\title{
Coexisting Pneumocystis carinii pneumonia, cytomegalovirus pneumonitis and salmonellosis in systemic lupus erythematosus
}

\author{
K H Leong; M L Boey, P H Feng
}

\begin{abstract}
Infection with opportunistic organisms, either singly or in combination, is known to occur in immunocompromised patients. A patient with systemic lupus erythematosus who developed Pneumocystis carinii pneumonia, cytomegalovirus pneumonitis, and salmonellosis is reported. She responded to early treatment with intravenous trimethoprim-sulphamethoxazole $(20 \mathrm{mg} / \mathrm{kg})$.
\end{abstract}

Pneumocystis carinii pneumonia occurs in immunocompromised patients ${ }^{1}$ and although treatable, is associated with a high death rate. Definitive diagnosis requires the demonstration of the organism in specimens obtained by bronchoalveolar lavage, transbronchial biopsy, or open lung biopsy. Pneumocystis carinii pneumonia can coexist with cytomegalovirus pneumonitis in the immunocompromised host and is associated with a poor prognosis. ${ }^{2}$

Another infection which often occurs in immunocompromised patients is salmonellosis. ${ }^{3}$ Bacteraemia is associated with concomitant infection in the bones, joints, the gut and, less commonly, the lung.

We report a patient with systemic lupus erythematosus who developed salmonellosis and a cavitating pneumonia in the presence of Pneumocystis carinii and cytomegalovirus.

\section{Case history}

A diagnosis of systemic lupus erythematosus was made in May 1985 in a 19 year old Eurasian girl from Indonesia when she developed fever, malar rash, and alopecia. She had a positive antinuclear antibody test, LE cell test, and hypocomplementaemia. Between 1986 and 1988 she experienced several flares of her illness marked by arthritis, cutaneous vasculitis, thrombocytopenia, and cerebral lupus. In October 1988 she developed nephrotic syndrome with a raised serum creatinine of 248 $\mu \mathrm{mol} / \mathrm{l}$ (normal $88-133 \mu \mathrm{mol} / \mathrm{l}$ ), and a reduced creatinine clearance of $33 \mathrm{ml} / \mathrm{min}$. A renal biopsy showed diffuse, proliferative nephritis (WHO class IV). High dose oral corticosteroids, oral cyclophosphamide, and pulse intravenous cyclophosphamide were given.

She developed pain in the right hip in February 1989. Clinical examination showed a young woman with Cushingoid facies, a temperature of $37 \cdot 8^{\circ} \mathrm{C}$, tinea corporis, and proximal myopathy. Blood pressure was normal. Her right hip was tender and the range of movements was markedly diminished. Laboratory values showed haemoglobin $82 \mathrm{~g} / \mathrm{l}$, white cell count $6.5 \times 10^{9} / 1$, platelets $150 \times 10^{9} / 1$. The erythrocyte sedimentation rate was $104 \mathrm{~mm}$ in the first hour. Radiography and a bone scan showed avascular necrosis of both femoral heads. A chest $x$ ray was normal. Two specimens of blood cultures grew salmonella group B, which showed in vitro sensitivity to aztreonam (Azactam). Aztreonam was given and the fever settled.

On the 13th day in hospital her temperature reached $38^{\circ} \mathrm{C}$. Repeat chest radiography showed a cavity in the right upper zone (fig 1). Two specimens of sputum were negative for acid fast bacilli. Antituberculous treatment (rifampicin, isoniazid, and ethambutol) was started empirically because pulmonary tuberculosis is endemic in Indonesia. She remained febrile and developed a non-productive cough and breathlessness. Blood cultures were negative. Serial chest radiographs showed multiple opacities over the entire lung fields (fig 2). Arterial blood gas examination showed pH 7.39, $\mathrm{PCO}_{2} 29 \cdot 2 \mathrm{mmHg}, \mathrm{PO}_{2} 67 \cdot 9$

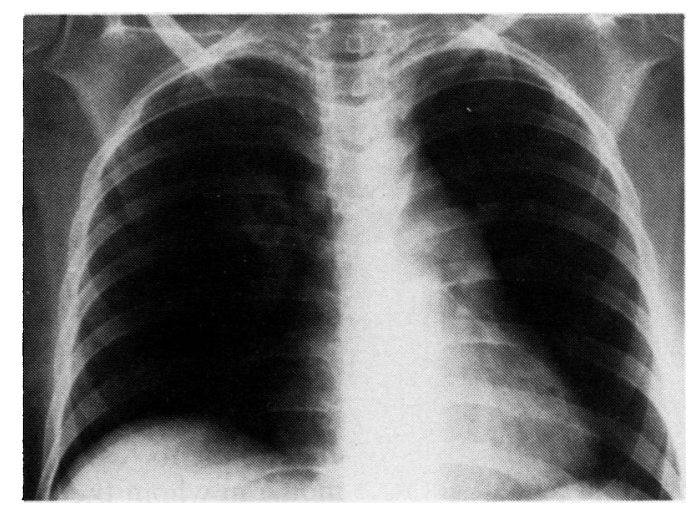

Figure 1 Cavity in the right upper zone.

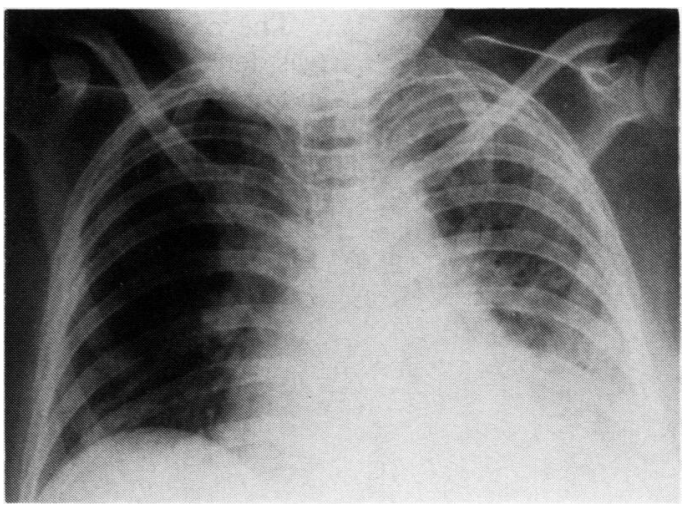

Figure 2 Bilateral lung infiltrates.
Dr Leong.

Accepted for publication 18 October 1990 
$\mathrm{mmHg}$, serum bicarbonate $19 \cdot 7 \mathrm{mmol} / \mathrm{l}$, and oxygen saturation $92.9 \%$. C reactive protein was $189 \mathrm{U} / \mathrm{ml}$ (normal 0-10), serum immunoglobulins were normal, and the T4/T8 ratio was 0.33 (normal $1 \cdot 0-2 \cdot 2$ ). HIV serology by enzyme linked immunosorbent assay (ELISA) was negative.

An open lung biopsy was performed eight days after the start of antituberculous drug treatment. Histology was consistent with Pneumocystis carinii pneumonia, and viral isolation by immunofluorescent techniques showed the presence of cytomegalovirus and cytopathic effects in the lung. Special stains failed to show evidence of fungi or acid fast bacilli. Although the biopsy specimen did not show salmonella pneumonia, cultures from the chest tube drainage fluid were positive for salmonella group B. Cytomegalovirus was also isolated from the urine.

Treatment with intravenous trimethoprimsulphamethoxazole $(20 \mathrm{mg} / \mathrm{kg})$ was immediately started and continued for three weeks. The dose of prednisolone was tapered to reduce the severity of the cytomegalovirus pneumonitis. The pulmonary shadows disappeared and the patient remained well four weeks after discharge from hospital.

\section{Discussion}

Infection has been acknowledged as a major factor in the morbidity and mortality of patients with systemic lupus erythematosus. ${ }^{4}$ The presence of active lupus and corticosteroid treatment increase the risk of bacterial, viral, and fungal invasion. Pulmonary infections may present with clinical and radiological features which are similar to lupus pneumonitis, making diagnosis difficult.

Pneumocystis carinii pneumonia was confirmed in our patient by demonstration of the organism in specimens obtained from the lung. The initial radiographic appearance of a cavity in Pneumocystis carinii pneumonia is unusual. Typical $x$ ray changes are bilateral interstitial or alveolar infiltrates, which often evolve from a perihilar location to a more diffuse and extensive pattern within three or four days. ${ }^{5}$ Cavitating lesions are uncommon but have been reported to simulate tuberculosis. Milligan et al described eight patients with Pneumocystis carinii pneumonia in whom the initial diagnosis of pulmonary tuberculosis was made and antituberculosis treatment started. ${ }^{6}$

The presence of cytomegalovirus in the lung was detected by positive cultures from bronchoscopy specimens and may be due to viral shedding in immunocompromised patients.
Isolation of the virus from lung tissue by an immunofluorescent technique suggested active pneumonitis, however. This was further confirmed by the presence of cytopathic effects on histology.

An increased infection rate for salmonella in systemic lupus erythematosus has been reported. ${ }^{7}$ Systemic lupus erythematosus and salmonellosis share similar clinical and laboratory denominators, such as fever, rash, pleurisy, synovitis, glomerulonephritis, leucopenia, and circulating immune complexes. ${ }^{7}$ Diagnosis may thus be delayed. Several factors may help explain the severity of the infection in our patient. Salmonella is an intracellular organism that survives despite the presence of humoral antibodies and systemic antibodies. In humans salmonellosis is unusually severe in patients with impaired $T$ cell function. These abnormalities include lymphopenia, a decreased number of $T$ helper cells, and a decreased ratio of $\mathrm{T}$ helper to $\mathrm{T}$ suppressor lymphocytes. ${ }^{8}$

We conclude that in patients with systemic lupus erythematosus who are severely immunocompromised, multiple opportunistic infections can coexist. As far as we know, coexisting Pneumocystis carinii pneumonia, cytomegalovirus pneumonitis, and salmonellosis in systemic lupus erythematosus has not been reported before. The clinical and radiographic features are non-specific. Commonly an aggressive diagnostic approach involving invasive procedures is required in an immunocompromised host with unexplained pulmonary infiltrates. Early institution of appropriate antimicrobial drugs will enhance the chances of recovery in such patients.

We thank Dr David Allen, Tan Tock Seng Hospital, for his invaluable comments.

1 Kovacs J A, Hiemenz J W, Macher A M, et al. Pneumocystis carinii pneumonia-a comparison between patients with the acquired immunodeficiency syndrome and patients with other

2 Jacobsen M A, Mills J. Serious cytomegalovirus disease in the acquired immunodeficiency syndrome. Ann Intern Med acquired immunodef

3 Saphra I, Winter I W Clinical manifestations of salmonellosis in man. $N$ Engl $\mathcal{Y}$ Med 1957; 256: 1128-34.

4 Staples P J, Gerding D W, Decker J L, Gordon R S. Incidence of infection in SLE. Arthritis Rheum 1974; 17: $1-10$.

5 DeLorenzo L J, Chin T H, Maguire G P, Stone D J. Roentgenographic patterns of Pneumocystis carinil pneumonia in 104 patients with AIDS. Chest 1987; 91: pneum.

6 Milligan S A, Stulbarg S S, Gamsu G, Golden J A. Pneumocystis carinii pneumonia radiographically simulating tuberculosis. Am Rev Respir Dis 1985; 132: 1124-6.

7 Lovy M R, Ryan P F J, Hughes G R V. Concurrent SLE and salmonellosis. $\mathcal{I}$ R heumatol 1981; 8: 605-12.

8 Jacobs J L, Gold J W M, Murray H W, Roberts R B Armstrong $\mathrm{D}$. Salmonella infections in patients with AIDS Ann Intern Med 1985; 102: 186-8. 